Pacific Journal of Mathematic 


\title{
SOME REMARKS ON HARMONIC MEASURE IN SPACE
}

\author{
William P. ZiEmer
}

\begin{abstract}
The purpose of this paper is to examine the relationship between harmonic measure and $n-1$ dimensional Hausdorff measure for a class of domains in $R^{n}$ with irregular boundaries. It is shown for these domains that harmonic measure and Hausdorff measure have the same null sets.
\end{abstract}

This investigation was motivated in part by the work of Hunt and Wheeden, [5], [6]. In these papers they consider Lipschitz domains, that is, domains whose boundaries are locally representable by graphs of Lipschitz functions. One of their main results is that a positive harmonic function defined on a Lipschitz domain has a nontangential limit at all points of the boundary except possibly those that belong to a set of harmonic measure zero. In the classical case where the domain is taken to be the half-space of $R^{n}$, the nontangential limit is known to exist at $H^{n-1}$ almost every point of the boundary, c.f., [2], [3]. We will show that for domains $\Omega$ satisfying a geometric measure theoretic condition, $H^{n-1}$ (restricted to the boundary of $\Omega$ ) and harmonic measure have the same null sets. Therefore, for these domains, the results of Hunt and Wheeden will represent a generalization of the classical case.

By use of the conformal mapping theorem it is not difficult to prove, for a domain in $R^{2}$ whose boundary is a simple closed rectifiable curve, that harmonic measure and $H^{1}$ measure have the same null sets. In $\S 4$ it will be shown that the analog of this does not hold in $R^{3}$. We give an example of a topological 2-sphere whose boundary has finite $H^{2}$ measure and has a tangent plane at each point, but for which $H^{2}$ measure is not absolutely continuous with respect to harmonic measure.

2. Preliminaries, Let $\Omega$ be a bounded open subset of $R^{n}$ and consider the Banach space $C(\partial \Omega)$, the space of continuous functions on the compact set $\partial \Omega$ with the norm given by $\sup \{|f(y)|: y \in \partial \Omega\}$, $f \in C(\partial \Omega)$. For each $x \in \Omega$, let $\lambda_{x}: C(\partial \Omega) \rightarrow R^{1}$ be the bounded linear functional defined by $\lambda_{x}(f)=u_{f}(x)$, where $u_{f}$ is the harmonic function corresponding to the boundary values $f$. Hence, there is a unit measure $\mu_{x}$ on $\partial \Omega$ called harmonic measure, such that

$$
\lambda_{x}(f)=u_{f}(x)=\int f d \mu_{x},
$$

$f \in C(\partial \Omega)$. If $G$ is a component of $\Omega$, the class of Borel subsets of 
$\partial \Omega$ of $\mu_{x}$ measure zero is independent of $x \in \Omega$. Moreover, the class of $\mu_{x}$ integrable functions is also independent of $x$.

The domains under consideration in this paper are contained in the class of sets of finite perimeter. A measurable set $E \subset R^{n}$ is said to have finite perimeter if the gradient of its characteristic function, when taken in the sense of distributions, is a totally finite vectorvalued measure. A unit vector $n$ is called the exterior normal (in measure theoretic sense) to $E$ at $y$ if the symmetric difference of $E$ and the half-space

$$
R^{n} \cap\{x:(x-y) \cdot n<0\}
$$

has density 0 at $y$. The set of points $y$ where $n=n(y)$ exists is called the reduced boundary of $E$ and is denoted by $\partial^{*} E$. If $E$ has finite perimeter, then $H^{n-1}\left(\partial^{*} E\right)<\infty$ and

$$
\int_{E} \operatorname{div} \zeta d L_{n}(x)=\int_{\partial^{*} L} \zeta(y) \cdot n(y) d H^{n-1}(y)
$$

for every smooth vector field $\zeta$. Here, $L_{n}$ denotes $n$-dimensional Lebesgue measure. Proofs of these facts concerning sets of finite perimeter can be found in [4].

Definition 2.1. For a measurable set $E \subset R^{n}, B \subset \partial E$, and $x \in R^{n}$, let the variation of $B$ at $x$ be defined by

$$
v(B, x)=\int_{\hat{\partial}^{*} \cap B} \frac{|n(y) \cdot(y-x)|}{|y-x|^{n}} d H^{n-1}(y) .
$$

If $v(B, x)<\infty$, then the following is meaningful:

$$
s(B, x)=\int_{\partial^{*} E \cap B} \frac{n(y) \cdot(y-x)}{|x-y|^{n}} d H^{n-1}(y) .
$$

In the event $E$ is a domain with smooth boundary, then $v(\partial E, x)$ can be regarded as the area of the radial projection

$$
p_{x}: \partial E \longrightarrow S^{n-1},
$$

and $s(\partial E, x)$ reduces to the notion of the solid angle.

By means of the Gauss-Green Theorem as given in (1), it can be easily verified that in case $E$ is a bounded open set with finite perimeter, then

$$
\begin{array}{ll}
s(\partial E, x)=\omega_{n-1}, & x \in E \\
s(\partial E, x)=0, & x \in R^{n}-\bar{E} .
\end{array}
$$

Where $\omega_{n-1}$ is the $H^{n-1}$ measure of the unit sphere in $R^{n}$.

Sets for which $v(\partial E, x)$ is a bounded function were investigated 
by Kral, [7], for the purpose of giving a geometric meaning to the normal derivative (taken in the sense of distributions) of the Newtonian potential.

3. Domains with boundaries having finite variation. Let $\Omega \subset R^{n}$ be an open, bounded, connected set, with $\partial$ (int $\left.\Omega\right)=\partial\left(R^{n}\right.$-Clos $\Omega$ ) and let $p \in \Omega$. Then the Green's function for $\Omega$ can be written as

$$
G(p, x)=I_{2}^{*} \delta(p)-I_{2}^{*} \mu_{p}
$$

where $I_{2}(x)=\left((n-2) \omega_{n-1}\right)^{-1}|x|^{2-n}$ and $\delta(p)$ denotes the unit measure concentrated at $p$. For $p$ fixed in $\Omega, G_{p}(x)=G(p, x)$ can be defined for all $x \notin \Omega$ and consequently if $\varphi \in C^{\infty}\left(R^{n}\right)$ with $p \notin \operatorname{spt} \varphi$,

$$
\Delta G_{p}(\varphi)=\mu_{p}(\varphi),
$$

where $\Delta$ is the Laplacian taken in the sense of distributions. On the other hand, if $\Omega_{k}$ is a sequence of domains with smooth boundaries invading $\Omega$, we have

$$
\begin{aligned}
\Delta G_{p}(\varphi) & =\int_{\Omega} G_{p} \Delta \varphi d L_{n}=\lim _{k \rightarrow \infty} \int_{\Omega_{k}} G_{p} \Delta \varphi d L_{n} \\
& =-\lim _{k \rightarrow \infty} \int_{\partial \Omega_{k}} \varphi \nabla G_{p} \cdot n d H^{n-1} \\
& =-\lim _{k \rightarrow \infty} \int_{\Omega_{k}} \nabla \varphi \cdot \nabla G_{p} d L_{n} \\
& =-\int_{\Omega} \nabla \varphi \cdot \nabla G_{p} d L_{n} .
\end{aligned}
$$

Therefore,

$$
\int_{\Omega} \nabla G_{p} \cdot \nabla \varphi=-\mu_{p}(\varphi)
$$

whenever $p \notin \operatorname{spt} \varphi$. If $h$ is a harmonic function in $\Omega$ whose gradient is integrable over $\Omega$, then the generalized normal derivative (using the exterior normal) of $h$ is defined by

$$
N h(\phi)=\int_{\Omega} \nabla h \cdot \nabla \varphi d L_{n}
$$

for all $\varphi \in C_{0}^{\infty}\left(R^{n}\right)$. We shall take the generalized normal derivative of the right side of (2).

First, it is elementary that

$$
N\left(I_{2}^{*} \delta(p)\right)(\varphi)=-1 / \omega_{n-1} \int_{\partial * \Omega} n(y) \cdot \frac{(y-p)}{|y-p|^{n}} \varphi(y) d H^{n-1}(y),
$$

and as $p \in \Omega, N\left(I_{2}^{*} \delta(p)\right)$ is a measure. 
Now in order to consider $N\left(I_{2}^{*} \mu_{p}\right)$ we impose the additional hypothesis

$$
\int v(\partial \Omega, y) d \mu_{p}(y)<\infty .
$$

Recall that this condition is independent of $p$. For any $y \in \partial \Omega$, observe that

$$
N\left(I_{2} * \delta(y)\right)(\varphi)=1 / \omega_{n-1} \int_{\Omega} \nabla \varphi(x) \cdot \frac{(y-x)}{|y-x|^{n}} d L_{n}(x) .
$$

Also, an application of Fubini's Theorem provides

$$
N\left(I_{2}^{*} \mu_{p}\right)(\varphi)=\int N\left(I_{2}^{*} \delta(y)\right)(\varphi) d \mu_{p}(y) .
$$

In [7], Kral investigated conditions under which $N\left(I_{2}^{*} \mu\right)$ is a measure whenever $\mu$ is a measure supported by $\partial \Omega$. For this purpose he proved

$$
\begin{aligned}
& \sup \left\{\int_{\Omega} \nabla \phi(x) \cdot \frac{y-x}{|y-x|^{n}} d L_{n}(x): \varphi \in C_{0}^{\infty}\left(R^{n}\right),|\varphi| \leqq 1\right\} \\
& \quad \leqq \omega_{n-1}+v(\partial \Omega, y) .
\end{aligned}
$$

Therefore, in particular, it follows from (4) and (5) that $N\left(I_{2}^{*} \mu_{p}\right)$ is a measure. In view of the fact that $\Omega$ is assumed to be of finite perimeter, it follows from the definition of the measure theoretic exterior normal that the Lebesgue $n$-dimensional density of $\Omega$ is equal to $1 / 2$ at $H^{n-1}$ almost all points of $\partial^{*} \Omega$. This allows us to conclude from [7, Lemma 3.2] that

$$
N\left(I_{2} * \delta(y)\right)(f)=\frac{f(y)}{2}-\frac{1}{\omega_{n-1}} \int_{\partial * \Omega} f(x) n(x) \cdot \frac{(x-y)}{|x-y|^{n}} d H^{n-1}(x)
$$

whenever $f$ is a bounded Baire function on $\partial \Omega$. Thus, if $E \subset \partial \Omega$ is a Borel set, it now follows from (6) that

$$
N\left(I_{2}^{*} \mu_{p}\right)(E)=\mu_{p}(E) / 2-1 / \omega_{n-1} \int s(E, y) d \mu_{p}(y) .
$$

From (3), (2), and (7) we obtain

$$
-\mu_{p}(E)=-1 / \omega_{n-1} s(E, p)-\left[\mu_{p}(E) / 2-1 / \omega_{n-1} \int s(E, y) d \mu_{p}(y)\right]
$$

or

$$
\omega_{n-1} / 2 \mu_{p}(E)=s(E, p)-\int s(E, y) d \mu_{p}(y)
$$


Thus, in summary, we have the following.

THEOREM 3.2. Let $\Omega$ be a bounded, open, connected set of finite perimeter with $\partial(\operatorname{int} \Omega)=\partial\left(R^{n}-\operatorname{Clos} \Omega\right)$. If $p \in \Omega$ and

$$
\int v(\partial \Omega, y) d \mu_{p}(y)<\infty,
$$

then

$$
\frac{\omega_{n-1}}{2} \mu_{p}(E)=s(E, p)-\int s(E, y) d \mu_{p}(y)
$$

for every Borel set $E \subset \partial \Omega$.

CoROLlaRY 3.3. If $E \subset \partial \Omega$ is a Borel set with $H^{n-1}\left(\partial^{*} \Omega \cap E\right)=0$, then $\mu_{p}(E)=0$.

The proof is obvious since $s(E, x)=0$ whenever $H^{n-1}\left(\partial^{*} \Omega \cap E\right)=0$ and $x \in R^{n}$.

We will now find conditions under which $H^{n-1}$ restricted to $\partial^{*} \Omega$ is absolutely continuous with respect to harmonic measure $\mu_{p}$. To this end we first establish

LEMMA 3.4. Suppose $\Omega$ is a set of finite perimeter and let $E \subset \partial^{*} \Omega$ be a Borel set of positive $H^{n-1}$ measure. There is a set $F \subset E$ with $H^{n-1}(E-F)=0$ satisfying the following conditions: if $\varepsilon>0, y \in F$, and if $p \in \Omega$ is on the interior normal to $\Omega$ at $y$, then there is a set $F_{p} \subset F$ containing $y$ such that

$$
\lim _{p \rightarrow y}\left(\operatorname{diam} F_{p}\right)=0
$$

and

$$
\lim _{p \rightarrow y} s\left(F_{p}, p\right)>\omega_{n-1} / 2-\varepsilon
$$

Proof. We refer the reader to Chapters 3 and 4 of [4] for proofs of the following facts. The set $\partial^{*} \Omega$ is $\left(H^{n-1}, n-1\right)$ rectifiable and therefore, there is a countable number of class $C^{1}$ manifolds of dimension $n-1, M_{i}$, such that

$$
H^{n-1}\left(\partial^{*} \Omega-\bigcup_{i \geqq 1} M_{i}\right)=0 .
$$

Moreover, for $H^{n-1}$ a.e. $y \in \partial^{*} \Omega$, the exterior normal to $\Omega$ at $y$ is orthogonal to the tangent plane of some $M_{i}$ at $y$. Finally, for $H^{n-1}$ a.e. $y \in E$, 


$$
\lim _{r \rightarrow 0} H^{n-1}\left(B(y, r) \cap E \cap M_{2}\right) / \alpha(n-1) r^{n-1}=1
$$

for some $M_{\imath}$. Here $\alpha(n-1)$ denotes the volume of the unit ball in $R^{n-1}$ and $B(y, r)$ is the $n$-ball of radius $r$ centered at $y$. Let $F$ denote the complement in $E$ of the union of the three $H^{n-1}$ null sets just described.

Now select $y \in F$ and $\varepsilon>0$. Then $y \in M_{\imath}$ for some $i$. For convenience, call $M_{i}=M$, take $y=0 \in R^{n}$, and assume the tangent plane to $M$ at 0 is $R^{n-1}$. Let $n$ denote the exterior normal at $y=0$ and for points $p$ on the interior normal at $y=0$, write $p=\left(0, v_{p}\right)$, $0 \in R^{n-1}, v_{p}<0$. Let $\eta=\eta(\varepsilon)$ be a number to be determined below and consider the cone

$$
C=\{x: n \cdot x>\eta|x|\} .
$$

For $x \in R^{n}$, write $x=(u, v), u \in R^{n-1}, v \in R^{1}$ and let

$$
C_{p}=\left\{(u, v): v=w+v_{p},(u, w) \in C\right\},
$$

the cone with vertex at $p$. Since $M$ is a manifold of class $C^{1}$, it is clear that for $\eta$ sufficiently small,

$$
\lim _{p \rightarrow 0} s\left(M_{p}, p\right)>\omega_{n-1} / 2-\varepsilon,
$$

where $M_{p}=M \cap C_{p}$. Let $A=M-F, F_{p}=F \cap C_{p}$, and $A_{p}=A \cap C_{p}$. Then

$$
\left|s\left(A_{p}, p\right)\right| \leqq \int_{A_{p}}|y-p|^{1-n} d H^{n-1}(y) .
$$

If $r_{p}=\sup \left\{|y|: y \in A_{p}\right\}$, then from (7)

$$
\lim _{p \rightarrow 0} H^{n-1}\left(A_{p}\right) \cdot r_{p}^{1-n}=0 \text {. }
$$

Furthermore, since $A$ is a subset of a class $C^{1}$ manifold, there is a constant $K$ such that $|y-p| \geqq K r_{p}$ whenever $y \in A_{p}$ and $|p|$ is sufficiently small. The conclusion of the lemma now follows from (8), (9), and (10).

In the following theorem, a condition is given under which $H^{n-1}$ restricted to $\partial^{*} \Omega$ is absolutely continuous with respect to harmonic measure. This condition is similar, although weaker, to the one introduced by $\mathrm{Kral}[7, \S 3]$ to determine the Fredholm radius of an operator associated with the classical double layer potential.

THEOREM 3.5. Let $\Omega$ be an open set satisfying the conditions of Theorem 3.2. In addition to the hypotheses of Theorem 3.2, assume that for $H^{n-1}$ a.e. $y_{0} \in \partial^{*} \Omega$, there exists a positive number $r=r\left(y_{0}\right)$ such that 


$$
\lim _{\substack{y \rightarrow y_{0} \\ y \in \partial * \Omega}} \sup v[B(y, r) \cap \partial \Omega, y]<\omega_{n-1} / 2 .
$$

Then $H^{n-1}\left(E \cap \partial^{*} \Omega\right)=0$ whenever $E \subset \partial \Omega$ is a Borel set with $\mu_{p}(E)=0$.

Proof. Let $E \subset \partial \Omega$ be a Borel set with $\mu_{p}(E)=0$ and assume that $H^{n-1}\left(E \cap \partial^{*} \Omega\right)>0$. Let $F \subset E$ be the set associated with $E$ as given by Lemma 3.4, and select $y_{0} \in F$ that satisfies the hypotheses of our theorem. Then, with every $\varepsilon>0$, there is a positive number $t<r\left(y_{0}\right)$ such that

$$
v[B(y, t) \cap \partial \Omega, y]<\omega_{n-1} / 2-\varepsilon .
$$

As $\mu_{p}(E)=\mu_{p}(F)=0$, it follows from Theorem 3.2 that

$$
s(F, p)=\int s(F, y) d \mu_{p}(y) .
$$

Now let $p$ approach $y_{0}$ along the interior normal and observe that Lemma 3.4 implies $F_{p} \subset B\left(y_{0}, t / 4\right)$ for $\left|p-y_{0}\right|$ sufficiently small. Therefore, it follows from (11) that

$$
\left|s\left(F_{p}, y\right)\right|<\omega_{n-1} / 2-\varepsilon
$$

for all $y \in B\left(y_{0}, t / 2\right)$. Moreover, $\left|s\left(F_{p}, y\right)\right| \rightarrow 0$ uniformly on $\partial \Omega-$ $B\left(y_{0}, t / 2\right)$ as $\left|p-y_{0}\right| \rightarrow 0$. In view of (12) and Lemma 3.4, this produces a contradiction. Therefore, $H^{n-1}\left(E \cap \partial^{*} \Omega\right)=0$.

4. An example. In $\S 3$ we imposed certain regularity conditions on $\partial \Omega$ in order to conclude that harmonic measure and $H^{n-1}$ restricted to $\partial^{*} \Omega$ have the same null sets. In $R^{2}$, harmonic measure and $H^{1}$ restricted to $\partial \Omega$ have the same null sets provided $\Omega$ is bounded by a simple closed curve of finite $H^{1}$ measure. In this section, we will show that the analog of this does not hold in $R^{3}$.

For the purpose of constructing this domain in $R^{3}$, we first consider a closed arc, $C$, in $R^{2}$ that occupies positive Lebesgue measure (or equivalently, $H^{2}$ measure) in $R^{2}$. It is not difficult to modify the standard Osgood construction of $C$ in such a way so as to produce a set, $S$, of points on $C$ of positive $H^{2}$ measure such that every point of $S$ cannot be joined to any point interior to $C$ by a rectifiable curve. Now let $g$ be function of class $C^{1}$ defined on the interior of $C$ with the properties

(i) $g>0$, (ii) $|\nabla g|$ is bounded, (iii) $g<\delta$, where $\delta(x)=$ distance from $x$ to $C$.

Define

$$
f(x)=\exp (-1 / g(x)), \quad x \in \text { interior } C,
$$


and let

$$
\Omega=\{(x, y): x \in \text { interior } C, 0 \leqq y<f(x)\} .
$$

Since $C$ is a closed arc, it follows that $\partial \Omega$ is a topological 2-sphere. Moreover, $\partial \Omega$ has a tangent plane at each of its points and $H^{2}(\partial \Omega)<\infty$. On the other hand, $S \subset \Omega$ is a set of positive $H^{2}$ measure with the property that each of its points cannot be joined to any point of $\Omega$ by a rectifiable curve. Consequently, a result of Brelot and Choquet [1] states that the harmonic measure of $S$ is zero.

In conclusion we would like to point out that there is no inclusion relationship between the class of smoothly bounded domains and the class of domains that satisfy the conditions of Theorem 3.5. However, if the normal of a smoothly bounded domain is locally Dini continuous, then Theorem 3.5 applies. We are indebted to Grant Welland for this observation.

To see this, let $f: U \rightarrow R^{1}$ be a function of class $C^{1}$ defined on the open set $U \subset R^{n-1}$. Let $S=\{(x, f(x)): x \in K\}$ where $K \subset U$ is a fixed compact set. It will suffice to prove there exists an $r>0$ such that $v(B(y, r) \cap S, y)$ can be made arbitrarily small for all $y \in S$. Fix $y_{0}=\left(x_{0}, f\left(x_{0}\right)\right) \in S$. Then for $r>0$,

$$
\begin{aligned}
v\left(B\left(y_{0}, r\right) \cap S, y\right)= & \int_{S} n(y) \cdot \frac{\left(y-y_{0}\right)}{\left|y-y_{0}\right|^{n}} d H^{n-1}(y) \\
= & \int_{K\left(|\nabla f(x)|^{2}+1\right)^{1 / 2}} \frac{\left(\nabla f(x), x_{0}, f(x)-f\left(x_{0}\right)\right)}{\left|y-y_{0}\right|^{n}} \\
& \times\left(|\nabla f(x)|^{2}+1\right)^{1 / 2} d L_{n-1}(x) \\
= & \int_{K} \frac{\left|\nabla f(x) \cdot\left(x-x_{0}\right)-\left[f(x)-f\left(x_{0}\right)\right]\right|}{\left|y-y_{0}\right|^{n}} d L_{n-1}(x) .
\end{aligned}
$$

Let $\rho=x-x_{0}$ and let points on the unit sphere in $R^{n-1}$ be denoted by $\theta$. Observe that there is a constant $M$ such that

$$
\left|x-x_{0}\right| \leqq\left|y-y_{0}\right| \leqq M\left|x-x_{0}\right|
$$

for all $x_{0}, x \in K$. Then, with $\rho \theta=x-x_{0}$,

$$
v\left(B\left(y_{0}, r\right) \cap S, y_{0}\right) \leqq N \iint\left\|\nabla f(x)|-| \nabla f\left(x_{\theta}\right)\right\| \frac{d_{0} \rho}{\rho} d \theta
$$

where $N$ is a constant and $x_{\theta}$ is some point on interval between $x_{0}$ and $x$. Hence, if the modulus of continuity of $|\nabla f|, \omega$, is required to satisfy

$$
\int_{0}^{1} \frac{\omega(r)}{r} d r<\infty
$$


then the conclusion obviously follows.

\section{REFERENCES}

1. M. Brelot and G. Choquet, Espaces et Lignes de Green, Ann. Inst. Fourier, 3 (1952), 199-263.

2. A. Calderon, On the behavior of harmonic functions at the boundary, Trans. Amer. Math. Soc., 68 (1950), 47-54.

3. L. Carleson, On the existence of boundary values for harmonic functions of several variables, Ark. Mat., 4 (1962), 393-399.

4. H. Federer, Geometric Measure Theory, Springer-Verlag, New York, 1969.

5. R. Hunt and R. Wheeden, On the boundary values of harmonic functions, Trans, Amer. Math. Soc., 132 (1968), 307-322.

6. - Positive harmonic functions on Lipschitz domains, Trans. Amer. Math. Soc., 147 (1970), 507-527.

7. J. Kral, The Fredholm method in potential theory, Trans. Amer. Math. Soc., 125 (1966), 511-547.

Received July 17, 1974. Research supported in part by a grant from the National Science Foundation.

INDIANA UNIVERSITY 



\section{PACIFIC JOURNAL OF MATHEMATICS}

\section{EDITORS}

RICHARD ARENS (Managing Editor)

University of California

Los Angeles, California 90024

\section{R. A. Beaumont \\ University of Washington \\ Seattle, Washington 98105}

\section{J. DugundJI}

Department of Mathematics

University of Southern California

Los Angeles, California 90007

D. Gilbarg and J. Milgram

Stanford University

Stanford, California 94305

\section{ASSOCIATE EDITORS}
E. F. BECKENBACH
B. H. NeumanN
F. WOLF
K. YOSHIDA

\section{SUPPORTING INSTITUTIONS}

UNIVERSITY OF BRITISH COLUMBIA
CALIFORNIA INSTITUTE OF TECHNOLOGY
UNIVERSITY OF CALIFORNIA
MONTANA STATE UNIVERSITY
UNIVERSITY OF NEVADA
NEW MEXICO STATE UNIVERSITY
OREGON STATE UNIVERSITY
UNIVERSITY OF OREGON
OSAKA UNIVERSITY

UNIVERSITY OF BRITISH COLUMBIA

UNIVERSITY OF CALIFORNIA

MONTANA STATE UNIVERSITY

NEW MEXICO STATE UNIVERSITY

OREGON STATE UNIVERSITY

OSAKA UNIVERSITY

\author{
UNIVERSITY OF SOUTHERN CALIFORNIA \\ STANFORD UNIVERSITY \\ UNIVERSITY OF TOKYO \\ UNIVERSITY OF UTAH \\ WASHINGTON STATE UNIVERSITY \\ UNIVERSITY OF WASHINGTON \\ AMERICAN MATHEMATICAL SOCIETY \\ NAVAL WEAPONS CENTER
}

The Supporting Institutions listed above contribute to the cost of publication of this Journal, but they are not owners or publishers and have no responsibility for its content or policies.

Mathematical papers intended for publication in the Pacific Journal of Mathematics should be in typed form or offset-reproduced, (not dittoed), double spaced with large margins. Underline Greek letters in red, German in green, and script in blue. The first paragraph or two must be capable of being used separately as a synopsis of the entire paper. Items of the bibliography should not be cited there unless absolutely necessary, in which case they must be identified by author and Journal, rather than by item number. Manuscripts, in triplicate, may be sent to any one of the editors. Please classify according to the scheme of Math. Reviews, Index to Vol. 39. All other communications should be addressed to the managing editor, or Elaine Barth, University of California, Los Angeles, California, 90024.

The Pacific Journal of Mathematics expects the author's institution to pay page charges, and reserves the right to delay publication for nonpayment of charges in case of financial emergency.

100 reprints are provided free for each article, only if page charges have been substantially paid. Additional copies may be obtained at cost in multiples of 50 .

The Pacific Journal of Mathematics is issued monthly as of January 1966. Regular subscription rate: $\$ 72.00$ a year (6 Vols., 12 issues). Special rate: $\$ 36.00$ a year to individual members of supporting institutions.

Subscriptions, orders for back numbers, and changes of address should be sent to Pacific Journal of Mathematics, 103 Highland Boulevard, Berkeley, California, 94708.

PUBLISHED BY PACIFIC JOURNAL OF MATHEMATICS, A NON-PROFIT CORPORATION

Printed at Kokusai Bunken Insatsusha (International Academic Printing Co., Ltd.), 270, 3-chome Totsuka-cho, Shinjuku-ku, Tokyo 160, Japan.

Copyright (C) 1973 by Pacific Journal of Mathematics Manufactured and first issued in Japan 


\section{Pacific Journal of Mathematics \\ Vol. 55, No. $2 \quad$ October, 1974}

Walter Allegretto, On the equivalence of two types of oscillation for elliptic

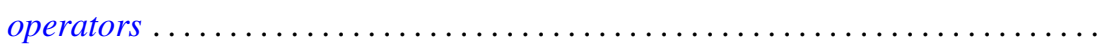

Edward Arthur Bertram, A density theorem on the number of conjugacy classes in

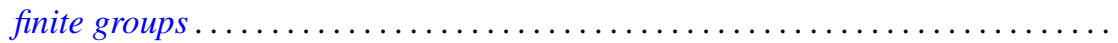

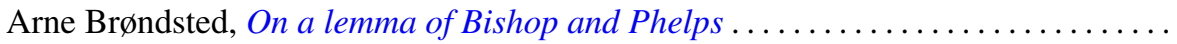

Jacob Burbea, Total positivity and reproducing kernels ..................

Ed Dubinsky, Linear Pincherle sequences . . . . . . . . . . . . . . . . . .

Benny Dan Evans, Cyclic amalgamations of residually finite groups .............

361

Barry J. Gardner and Patrick Noble Stewart, A "going down" theorem for certain

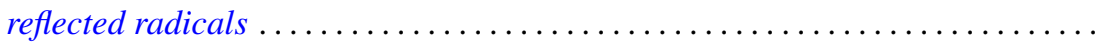

381

Jonathan Light Gross and Thomas William Tucker, Quotients of complete graphs:

revisiting the Heawood map-coloring problem ....................

Sav Roman Harasymiv, Groups of matrices acting on distribution spaces .........

Robert Winship Heath and David John Lutzer, Dugundji extension theorems for

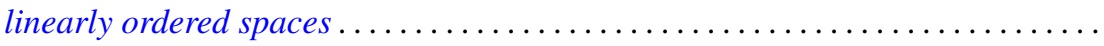

Chung-Wu Ho, Deforming p. l. homeomorphisms on a convex polygonal

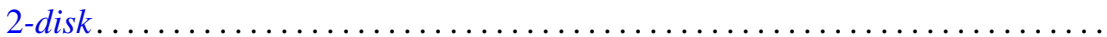

Richard Earl Hodel, Metrizability of topological spaces .................

Wilfried Imrich and Mark E. Watkins, On graphical regular representations of

cyclic extensions of groups .......................... 461

Jozef Krasinkiewicz, Remark on mappings not raising dimension of curves ..... . 479

Melven Robert Krom, Infinite games and special Baire space extensions . . . . . . 483

S. Leela, Stability of measure differential equations . . . . . . . . . . . . . . . . 489

M. H. Lim, Linear transformations on symmetric spaces . . . . . . . . . . . . . . . 499

Teng-Sun Liu, Arnoud C. M. van Rooij and Ju-Kwei Wang, On some group algebra modules related to Wiener's algebra $M_{1} \ldots \ldots \ldots \ldots \ldots \ldots \ldots \ldots \ldots \ldots \ldots$

Dale Wayne Myers, The back-and-forth isomorphism construction ............ 521

Donovan Harold Van Osdol, Extensions of sheaves of commutative algebras by

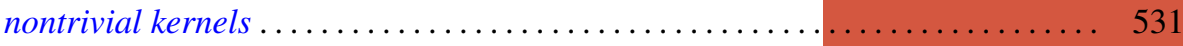

Alan Rahilly, Generalized Hall planes of even order ................... 543

Joylyn Newberry Reed, On completeness and semicompleteness of first countable

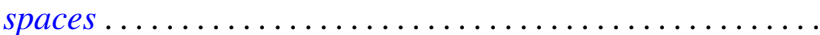

Alan Schwartz, Generalized convolutions and positive definite functions associated

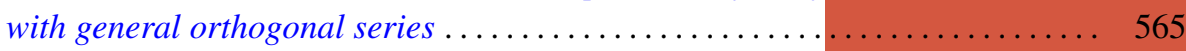

Thomas Jerome Scott, Monotonic permutations of chains . . . . . . . . . . . 583

Eivind Stensholt, An application of Steinberg's construction of twisted groups .... 595

Yasuji Takeuchi, On strongly radicial extensions . . . . ................. 619

William P. Ziemer, Some remarks on harmonic measure in space . . . . . . . . . . 629

John Grant, Corrections to: “Automorphisms definable by formulas” . . . . . . . . 639

Peter Michael Rosenthal, Corrections to: "On an inversion for the general

Mehler-Fock transform pair" ......................... 640

Carl Clifton Faith, Corrections to: "When are proper cyclics injective” . . . . . . 640 